\title{
ARTIGOS
}

\section{Evaluación de barreras vegetales en el manejo integrado de la mancha anular del papayo (PRSV-P) en Michoacán, México}

\author{
Patricia Rivas-Valencia ${ }^{1}$, Gustavo Mora-Aguilera ${ }^{1}$, Daniel Téliz-Ortiz $^{1}$, y Antonio Mora-Aguilera ${ }^{1}$
}

${ }^{1}$ Instituto de Fitosanidad, Colegio de Postgraduados, Km 35.5, Carr. México-Texcoco, Montecillo, Edo. de México, CP 56230, México. E-mail: morag@colpos.mx.

Autor para correspondência: Gustavo Mora-Aguilera

Data de chegada: 16/02/2005. Aceito para publicação em: 3/11/2007

1174

\section{RESUMEN}

Rivas-Valencia, P.; Mora-Aguilera, G.; Téliz-Ortiz, D.; Mora-Aguilera, A. Evaluación de barreras vegetales en el manejo integrado de la mancha anular del papayo en Michoacán, México. Summa Phytopathologica, v.34, n.4, p.307-312, 2008

El efecto de barreras vegetales como componente de un programa de manejo integrado (MI), se validó y adaptó en 1999 en Michoacán, México, para controlar la Mancha Anular del Papayo, enfermedad causada por el Papaya ringspot potyvirus type-P (PRSV-P). Se estableció un experimento en parcelas divididas con dos factores experimentales: barreras vegetales (Hibiscus sabdariffa), y componentes de MI: MI sin aspersión de citrolina (1.5\%) (MI-A), MI sin eliminación de plantas con síntomas iniciales de virosis antes de floración (MI-D) y MI. Las barreras vegetales sembradas 20 días antes del trasplante del papayo y el desplante retrasaron en 19 días el inicio del progreso de epidemias en el MI lo que resultó en una mayor producción (14.2\%) que el resto de tratamientos, aunque fue superado por MI-A en vigor ( $4 \%$ en diámetro de tallo). La citrolina fue fitotóxica, disminuyó el vigor de plantas (5.3\%) y no limitó significativamente el desarrollo de la enfermedad ya que la intensidad de las epidemias $\left(\mathrm{X}_{0}=47\right.$ días, $\mathrm{Y}_{\mathrm{f}}=84 \%$ y ABCPE $=3220 \%$ días $)$ fue similar al testigo. El uso de barreras vegetales por si sola aparentemente no es suficiente para la reducción de la incidencia y dispersión de la enfermedad. Los áfidos más abundantes, con reconocida capacidad transmisora del PRSV-P, fueron Aphis gossypii, A. nerii, A. spiraecola y Macrosiphum euphorbiae, los cuales representaron aproximadamente el $13 \%$ del total de áfidos capturados.

Palabras clave adicionales: Papaya ringspot potyvirus type-P, Maradol, epidemiología, barreras vegetales, desplante.

\section{ABSTRACT}

Rivas-Valencia, P.; Mora-Aguilera, G.; Téliz-Ortiz, D.; Mora-Aguilera, A. Evaluation of plant barriers in an integrated management of papayo ringspot in Michoacan, Mexico.Summa Phytopathologica, v.34, n.4, p.307-312, 2008

The effect of plant barriers as a component of an integrated management program (IM) was validated and adapted in 1999, in Michoacan, Mexico, to control papaya ringspot, caused by papaya ringspot potyvirus type-P (PRSV-P). A split-plot design was established with two experimental factors: plant barriers and components of IM: IM without oil sprinkling (IM-O), IM without plant rouging (IM-R), and complete IM. Plant barriers (Hibiscus sabdariffa), sowed 20 days before papaya transplanting, and plant rouging delayed the epidemics onset in 19 days thus IM resulted in the highest yield (14.2\%) than the rest of the treatments, but it was less effective than IM-O in vigor (4\% in stem diameter). Oil sprinkling was phytotoxic and caused a $5.3 \%$ vigor reduction. The oil effect on the disease progress was not clear, since the epidemic intensity (epidemic onset $X_{0}=47$ days after transplanting, final incidence $\mathrm{Y}_{\mathrm{f}}=81 \%$ and area under disease progress curve $\mathrm{ABCPE}=3220$ \%days) was similar to the control treatment. Plant barriers alone may not be sufficient to reduce disease incidence and spreading. The more abundant winged aphids known as PRSV-P vectors were Aphis gossypii, A. nerii, A. spiraecola and Macrosiphum euphorbia, which represented approximately $13 \%$ of the total captured aphids.

Keywords: Papaya ringspot potyvirus type-P, Maradol, epidemiology, plant barriers, rouging.

El papayo (Carica papaya L.) es una planta de origen americano (15) que se ha extendido por amplias regiones tropicales y subtropicales del mundo, con una producción significativa en Brasil, Tailandia, Nigeria, India, México e Indonesia (11). En México se cultivaron 22,451ha de papayo en 2007, principalmente en Chiapas, Michoacán, Veracruz y Tabasco (22). Las papayas cultivadas más extensivamente en México han sido la criolla o tipo "Cera" y Maradol; sin embargo, las variedades Maradol y hawaianas son más demandadas en el mercado de exportación. En 2007, México exportó 43,921 ton con ingresos por 243,844,319 dólares americanos y ubicó a este frutal en el sexto sitio entre las frutas tropicales y subtropicales mexicanas de exportación (11). 
La mancha anular del papayo causada por el Papaya ringspot potyvirus type-P (PRSV-P) es la principal enfermedad del cultivo de papayo en México $(1,3,5,7,10,23)$. Las plantas afectadas por el PRSV-P presentan síntomas de mosaico, deformación foliar, amarillamiento, manchas aceitosas en pecíolos, tallos y frutos. El síntoma típico es la formación de manchas anulares en el fruto y eventual deformación del mismo. Plantas con infecciones tempranas reducen severamente su crecimiento y puede anular su producción. Plantas adultas disminuyen su dosel y defolian exponiendo los frutos a quemaduras por el sol. El período productivo comercial se reduce apróximadamente a un año en México (1, 3, 10, 14, 23). El PRSV-P se transmite por áfidos (Homóptera: Aphididae), principalmente Aphis nerii, A. gossypii, A. spiraecola (A. citricola) y Macrosyphum euphorbiae en forma no persistente $(5,13,14,23)$.

Abundante información se ha generado en relación a la aplicación de métodos de control individuales para el PRSV-P en papayo: Control químico, cultural, físico, protección cruzada, variedades tolerantes, etc., sin embargo, el desarrollo de tecnologías de control del PRSV-P a través de estrategias focalizadas en el manejo integrado del patógeno o plaga (MIP) o manejo integral del cultivo (MIC) han sido escasas en el mundo. En contraste, en México, el Grupo Interdisciplinario e Interinstitucional del Papayo (GIIP) del Colegio de Postgraduados (CP) e Instituto Nacional de Investigaciones Forestales, Agrícolas y Pecuaria (INIFAP), han realizado investigaciones con el fin de generar propuestas tecnológicas de manejo integrado (MI) del cultivo para reducir las pérdidas ocasionadas por el PRSV-P, mediante: protección de viveros con mallas antiáfidos; uso de cultivos como barreras vegetales, e.g. maíz (Zea mays) sorgo (Sorgum sp.) y jamaica (Hibiscus sabdariffa L.); mayor densidad de plantación (2200-3117 plantas/ha${ }^{1}$ ); aspersión foliar de aceites minerales derivados del petróleo (citrolina o Safe-T-Side ${ }^{\circledR}$ al $1.5 \%$ ), y eliminación de plantas con síntomas de virosis desde el transplante y hasta inicio de floración $(2,5,7,10$, 18, 20, 24). En Michoacán se ha incrementado el área cultivada de papaya en los últimos años y los productores han adoptado empíricamente técnicas fitosanitarias y de producción, sin resultados satisfactorios. Los objetivos de este estudio en Michoacán fueron: a) evaluar y adaptar componentes técnicos individuales de MI generada en Veracruz, México: uso de barreras vegetales (Hibiscus sabdariffa), aspersión de citrolina (aceite mineral) al $1.5 \%$ y eliminación de plantas con síntomas iniciales de mancha anular, para reducir la incidencia y severidad causada por el PRSV-P e incrementar la productividad; b) caracterizar el progreso temporal de epidemias; y c) identificar y estimar la densidad estacional de especies de áfidos potencialmente transmisores del PRSV-P.

\section{MATERIALES Y MÉTODOS}

\section{Establecimiento del experimento}

Un experimento de papayo cv. Maradol se estableció en 1999, en El Chauz, Municipio de La Huacana, Michoacán, México, ubicado a $18^{\circ} 56^{\prime}$ latitud norte y $101^{\circ} 48^{\prime}$ longitud oeste, con clima tropical subhúmedo con lluvias en verano [Aw0(w)] y suelo andosol (8). El vivero se estableció en el municipio vecino de Antúnez, el 27 de abril de 1999 con semilla certificada de la variedad maradol. El manejo del vivero consistió de pregerminación, riegos manuales periódicos y fertilización. El vivero se cubrió con una malla plástica antiáfidos para evitar la transmisión temprana del PRSV-P. El transplante se efectúo el siete y ocho de junio del mismo año con plantas previamente podadas a una altura de $40 \mathrm{~cm}$. El suelo de tipo arcilloso con un $\mathrm{pH}$ de
7.4, se preparó mediante rastreo, barbecho y surcado cada $110 \mathrm{~cm}$ de separación. Cada seis surcos de papayo se intercalaron tres surcos de plantas de jamaica separadas $70 \mathrm{~cm}$ entre sí y sembrados 20 días antes del transplante del papayo. La técnica MI se ajustó y evaluó en un diseño de parcelas divididas con tres repeticiones y una densidad de 2200 plantas $\mathrm{ha}^{-1}$, en un área de $108 \times 75 \mathrm{~m}$. Las parcelas grandes correspondieron a tratamientos con y sin barreras vegetales de jamaica y las parcelas chicas (repeticiones experimentales con 60 plantas) correspondieron a los tratamientos: 1) testigo tradicional (TT), sin protección física de vivero, aspersión de citrolina y eliminación de plantas enfermas; 2) Manejo Integrado (MI), vivero protegido, aspersión de citrolina al $1.5 \%$ al envés de las hojas cada 10 días y eliminación de plantas con síntomas iniciales de virosis desde el transplante y hasta inicio de floración; 3) MI sin citrolina (MI-A), constituyó las prácticas del MI a excepción de la aspersión de citrolina; y 4) MI sin eliminación de plantas enfermas (MI-D), constituyó todas las prácticas del MI menos la eliminación de plantas con síntomas iniciales de virosis. La separación entre parcelas grandes y chicas fue de $3 \mathrm{~m}$ mediante surcos sin plantas. El manejo general del cultivo consistió en riegos por gravedad, eliminación de maleza en forma manual y química (glifosato dos litros en 100 litros de agua) y fertilización tradicional de NPK $(17: 17: 17)$ en una cantidad aproximada de $65 \mathrm{~g}$ planta $^{-1}$

\section{Medición de variables}

Se evaluaron seis plantas seleccionadas aleatoriamente por cada repetición experimental (24 plantas por tratamiento). Se realizaron siete evaluaciones a intervalos de 15 días. Las características evaluadas y las variables medidas fueron: a) vigor: altura de planta y diámetro de tallo, medido a $10 \mathrm{~cm}$ del suelo, con estadal y vernier respectivamente; número de hojas completamente expandidas; número, semiperímetro y diámetro de frutos; b) fenología: días a inicio de floración y fructificación y altura al primer fruto amarrado; c) sanidad viral: incidencia y severidad visual inducida por PRSV-P, estimada con una escala nominal arbitraria ( $1=$ planta sana, $2=$ mosaico tenue $o$ moteado amarillo ligero, $3=$ mosaico definido pero no generalizado en hojas, $4=$ mosaico severo generalizado en hojas y $5=$ mosaico severo con reducción de lámina foliar). De junio a diciembre de 1999, se realizaron colectas de áfidos cada tres días, en trampas de charolas plásticas de color amarillo, tipo Moericke con agua y jabón detergente en polvo (1 g) para eliminar la tensión superficial. La presencia del PRSV-P se confirmó mediante ELISA-DAS (Enzime Linked Inmuno Sorbent Assay; Agdia Inc.) en plantas de vivero previas al trasplante y en muestreos mensuales de ápices foliares tomados de 30 plantas y analizadas en tres muestras compuestas de 10 plantas por unidad experimental con un total de 720 plantas.

\section{Análisis de datos}

Las epidemias, construidas a partir de las evaluaciones periódicas de incidencia y severidad se caracterizaron de acuerdo con los parámetros $\mathrm{b}^{-1} \mathrm{y} \mathrm{c}$ del modelo de Weibull, que describe epidemias con forma e intensidad diversa, en contraposición a modelos no flexibles como el logístico y Gompertz (19). Los parámetros del área bajo la curva del progreso de la enfermedad (ABCPE), tiempo de inicio de la epidemia $\left(\mathrm{X}_{0}\right)$, incidencia final $\left(\mathrm{Y}_{\mathrm{f}}=\mathrm{Y}_{\max }\right)$ y severidad final $\left(\mathrm{S}_{\mathrm{f}}\right)$, se sometieron a un análisis de varianza para la comparación de epidemias entre tratamientos. La altura de planta y diámetro de tallo para cada tratamiento se caracterizó de acuerdo con el área bajo la curva del desarrollo de planta (ABCDP). Con datos del semiperímetro, diámetro $\mathrm{y}$ peso de frutos se generaron dos modelos lineales para estimar la 
Cuadro 1. Estimación del inverso del parámetro $\mathrm{b}\left(\mathrm{b}^{-1}\right)$ del modelo de Weibull y área bajo la curva del progreso de la enfermedad (ABCPE) e incidencia final $\left(\mathrm{Y}_{\mathrm{f}}\right)$ en el patosistema papayo-PRSV-P bajo algunas prácticas de manejo integrado Michoacán, México 1999.

\begin{tabular}{|c|c|c|c|c|c|c|}
\hline \multirow{2}{*}{ Tratamientos $^{x}$} & \multicolumn{3}{|c|}{ Con Barreras ${ }^{\mathrm{z}}$} & \multicolumn{3}{|c|}{ Sin Barreras ${ }^{\mathrm{z}}$} \\
\hline & $\mathbf{b}^{-1}$ & ABCPE & $Y_{f}=Y_{\max }$ & $b^{-1}$ & ABCPE & $Y_{f}=Y_{\text {max }}$ \\
\hline $\mathrm{T} \mathrm{T}$ & $0.0092 \mathrm{a}$ & $3828.26 \mathrm{a}$ & $83.32 \mathrm{a}$ & $0.0086 \mathrm{a}$ & $2521.21 \mathrm{a}$ & $94.43 \mathrm{a}$ \\
\hline MI & $0.0085 \mathrm{a}$ & $2937.73 \mathrm{a}$ & $72.20 \mathrm{a}$ & $0.0087 \mathrm{ab}$ & $3020.53 \mathrm{a}$ & $91.65 \mathrm{a}$ \\
\hline MI-D & $0.0084 \mathrm{a}$ & $3967.13 \mathrm{a}$ & $83.30 \mathrm{a}$ & $0.0084 \mathrm{a}$ & $2731.26 \mathrm{a}$ & $77.75 \mathrm{a}$ \\
\hline MI-A & $0.0088 \mathrm{a}$ & $3531.06 \mathrm{a}$ & $83.32 \mathrm{a}$ & $0.0093 \mathrm{~b}$ & $3228.25 \mathrm{a}$ & $87.02 \mathrm{a}$ \\
\hline
\end{tabular}

'TT: testigo tradicional regional; MI: Manejo integrado del papayo incluyendo barreras, citrolina y eliminación de plantas virosas; MI-A: MI sin citrolina; MI-D: MI sin eliminación de plantas con síntomas iniciales de virosis.

${ }^{\mathrm{z}}$ Tratamientos con la misma letra son estadísticamente similares. Tukey $(\mathrm{p}=0.05)$.

producción en los tratamientos con y sin barreras. Los análisis se realizaron por medio del programa estadístico SAS $\left(\mathrm{SAS}^{\circledR}\right.$ Institute, 1998) (21).

\section{RESULTADOS Y DISCUSIÓN}

\section{Caracterización de epidemias}

El progreso temporal de las diferentes epidemias del PRSV-P observadas en Michoacán fueron similares en forma e intensidad a las observadas en Veracruz $(12,13)$, siendo éstas de tipo sigmoidal (Figura 1). La ausencia de plantas enfermas por el PRSV-P, verificada mediante ELISA-DAS al momento del transplante, confirmó el efecto benéfico de la protección física del vivero contra áfidos potencialmente vectores. En contraste, se encontraron plantas enfermas durante los tres muestreos posteriores al transplante, es decir, a los 66 días después del transplante (DDT). El inicio de las epidemias $\left(\mathrm{X}_{0}\right)$ ocurrió, en general, 47 DDT. La fase exponencial de la epidemia ( $\mathrm{Y}_{\mathrm{e}}$ ) tuvo una duración de 55 a 74 DDT, la duración total $\left(\mathrm{T}_{\mathrm{f}}\right)$ tuvo un período de 87 DDT y la incidencia final $\left(\mathrm{Y}_{\mathrm{f}}=\mathrm{Y}_{\max }\right)$ fue de $100 \%$ y se alcanzó 134 DDT. En un estudio similar realizado en condiciones de temporal en Veracruz (13), se observó que los valores estimados para $\mathrm{X}_{\mathrm{o}}, \mathrm{T}_{\mathrm{f}}$ y $\mathrm{Y}_{\mathrm{f}}$ de 60 epidemias inducidas por PRSV-P fueron de 87, 209 DDT y $82 \%$, respectivamente. Las tasas de infección $\left(\mathrm{b}^{-1}\right)$ de 60 epidemias

Cuadro 2. Área bajo la curva del progreso de epidemia de mancha anular, diámetro de tallo, altura de planta y producción de papaya cv. Maradol (Carica papaya L.) bajo algunas prácticas de manejo integrado, Michoacán, México, 1999.

\begin{tabular}{|c|c|c|c|c|}
\hline Tratamientos ${ }^{a}$ & $\begin{array}{l}\mathrm{ABCPE}^{\mathrm{b}} \\
\left(\% \cdot \mathbf{d i ́}^{-1}\right)\end{array}$ & $\begin{array}{l}\text { ABCPDT }^{c} \\
\left(\% \cdot \mathrm{cm}^{-1}\right)\end{array}$ & $\begin{array}{l}\text { ABCPAP } \\
\left(\% \cdot \mathbf{c m}^{-1}\right)\end{array}$ & $\begin{array}{l}\text { Producción }^{\mathrm{e}} \\
\left(\operatorname{ton}_{h} \mathbf{h}^{-1}\right)\end{array}$ \\
\hline $\mathrm{T} \mathrm{T}$ & $3174.7 \mathrm{a}^{\mathrm{z}}$ & $605.4 b^{z}$ & $11043.3 b^{2}$ & $20.67 b^{2}$ \\
\hline MI & $2979.1 \mathrm{a}$ & $733.6 \mathrm{a}$ & $12594.4 \mathrm{a}$ & $27.98 \mathrm{a}$ \\
\hline MI-D & 3349.2 a & $723.0 \mathrm{a}$ & 12515.9 a & $24.76 \mathrm{ab}$ \\
\hline MI-A & 3379.7 a & $767.9 \mathrm{a}$ & 13085.9 a & $26.55 \mathrm{ab}$ \\
\hline Con Barreras & $3566.0 \mathrm{a}$ & 725.9 a & $12572.7 \mathrm{a}$ & $25.80 \mathrm{a}$ \\
\hline Sin Barreras & $2875.3 \mathrm{~b}$ & $689.1 \mathrm{a}$ & $12047.0 \mathrm{a}$ & $24.18 \mathrm{a}$ \\
\hline
\end{tabular}

${ }^{\text {aTT: }}$ testigo tradicional regional; MI: Manejo integrado del papayo incluyendo barreras, citrolina y eliminación de plantas virosas; MI-A: MI sin citrolina; MI-D: MI sin eliminación de plantas con síntomas iniciales de virosis.

${ }^{\mathrm{b}} \mathrm{ABCPE}$ : Area bajo la curva del progreso de la enfermedad.

${ }^{\mathrm{c}} \mathrm{ABCDT}$ : Promedio del área bajo la curva del desarrollo del diámetro de tallo.

${ }^{\mathrm{d}}$ ABCPAP: Promedio del área bajo la curva del desarrollo de altura de planta.

${ }^{\mathrm{z}}$ Tratamientos con la misma letra son estadísticamente similares. Tukey $(\mathrm{p}=0.05)$. tuvieron valores entre 0.0124 y 0.0021 (12), valores superiores a las tasas estimadas en Michoacán. La diferencia en los valores de $\mathrm{X}_{\mathrm{o}}, \mathrm{Y}_{\mathrm{f}} \mathrm{y}$ $\mathrm{T}_{\mathrm{f}}$ muestra la importancia de las condiciones climáticas contrastantes en las regiones estudiadas, esto es subhúmedo con lluvias en verano [Aw2(w)] vs el más seco del tipo subhúmedo [Aw0(w)] para Veracruz y Michoacán, México, respectivamente (8). En clima seco, se esperaría, una menor diversidad de hospedantes alternos del virus y de vectores potenciales, pero este no parece ser el caso. La explicación podría ser el régimen de riego, que permite la existencia de mayor diversidad de cultivos y malezas hospederas del PRSV-P fuera del período de lluvias, como se ha sugerido para otros patosistemas virales (17) y como ocurre durante la mayor parte del año en Michoacán. De 594 especimenes capturados, sólo 77 correspondieron a individuos alados potencialmente vectores del PRSV-P: Aphis gossypii (39 individuos), A. nerii (24 ind.), A. spiraecola (13 ind.) y Macrosiphum euphorbiae (1 ind.) (Figura 2). Estas mismas especies y Myzus persicae son responsables de la mayoría de las epidemias del PRSV-P en Veracruz. En este patosistema la densidad poblacional de A. spiraecola superó a las otras especies en más del $300 \%$. La ausencia de colonización de plantas de papayo por áfidos $(5,13)$ sugiere la importancia de las poblaciones aladas en la dispersión del virus. De acuerdo a resultados previos obtenidos con un modelo de pronóstico (13), los principales vectores en Veracruz fueron A. nerii, A. gossypii y M. persicae.

\section{Efectos de tratamientos en epidemias}

Las barreras vegetales interfirieron en la eficiencia de transmisión viral por áfidos, al retrasar significativamente el inicio y progreso de las epidemias em MI. Estos resultados confirmaron investigaciones realizadas por el GIIP (4) y Becerra-Leor et al. (3) en Veracruz. Aunque el inicio de las epidemias $\left(\mathrm{X}_{0}\right)$ ocurrió 47 DDT en tratamientos sin y con barreras (Figura 1), la intensidad de las epidemias, calculadas mediante el ABCPE, fue menor con presencia de barreras $(\mathrm{p}=0.05)$ (Cuadro 1). En contraste, la intensidad de las epidemias, estimadas mediante el inverso del parámetro $\mathrm{b}^{-1}$ del modelo de Weibull, entre ambos tratamientos, no fue estadísticamente significativa (Cuadro 1), sin embargo, numéricamente los valores observados fueron menores en el tratamiento con barreras. Es necesario notar que el efecto de barreras fue significativo aún cuando este tratamiento fue asignado a las parcelas grandes con la consiguiente pérdida de precisión para este factor en el análisis de varianza, lo que sugiere, que en trabajos futuros se asigne esta variable a parcelas chicas.

El efecto benéfico de las barreras podría mejorarse incorporando nuevos criterios de manejo como combinación de especies (e. g. maíz, sorgo forrajero, jamaica, etc.) y variedades de distinto ciclo y porte con siembras en fechas escalonadas para mejorar su efecto protectivo durante todo el ciclo productivo del papayo o durante las fases más críticas de daño del cultivo que coinciden con las etapas tempranas de 


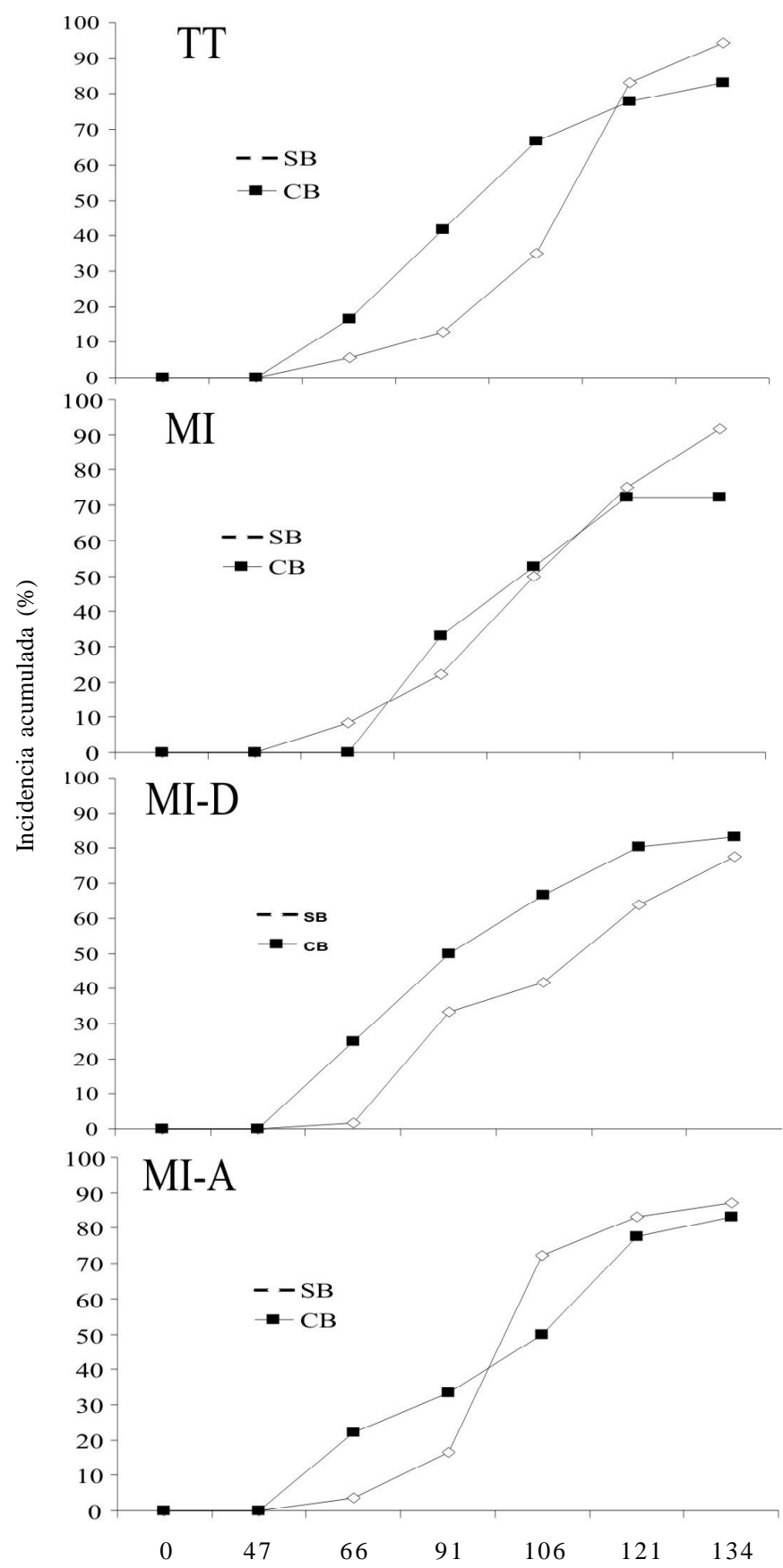

Días después del transplante (DDT)

Figura 1. Curvas del progreso de la enfermedad causada por el Papaya ringspot potyvirus type-P (PRSV-P) en papayo cv. Maradol en Michoacán, México 1999. CB: Plantación con barreras; SB: Plantación sin barreras; TT: testigo tradicional; MI: Manejo integrado de papayo; MI-A: MI sin citrolina; MI-D: MI sin eliminación de plantas con síntomas iniciales de virosis.

desarrollo. Las barreras de jamaica interfieren físicamente con el movimiento horizontal de áfidos potencialmente transmisores y la coloración rojiza de tallos, pecíolos y brácteas florales, ejercen un efecto repelente contra estos (3). En Michoacán, la jamaica protegió la plantación de papayo sólo por un período corto de tiempo, ya que fue superada en altura por el cultivo. Esta limitante también se acentúa por su ciclo corto de vida y a su crecimiento que es afectado por el

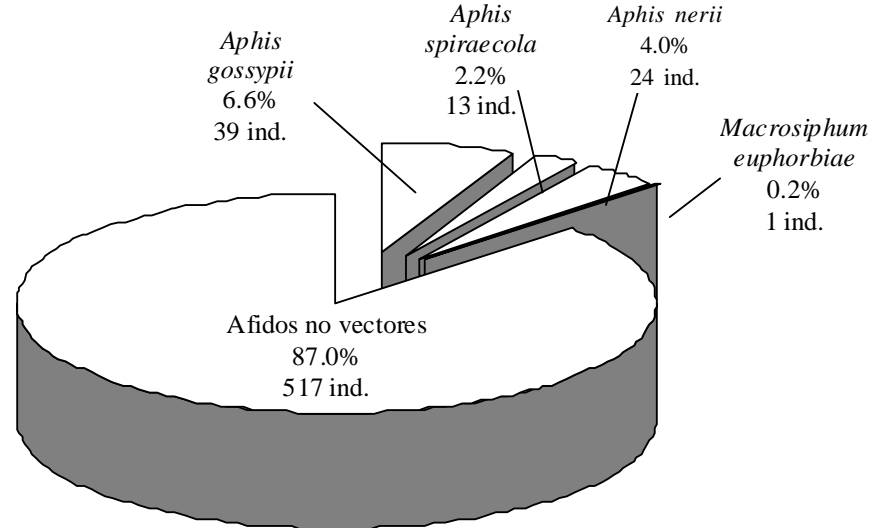

Figura 2. Áfidos totales alados capturados en trampas plásticas amarillas tipo Moericke en una parcela experimental de papayo cv. Maradol bajo algunas prácticas de manejo integrado en Michoacán, México, 1999. Período de captura: junio a diciembre, 1999. Cantidad total= 594; áfidos potencialmente vectores $=77$.

fotoperíodo. Los tratamientos evaluados como parcelas chicas (MI-D y MI-A), en general, no difirieron con el programa MI originalmente propuesto para Veracruz, aunque tampoco fueron distintos del TT en lo que respecta a los parámetros $\mathrm{b}^{-1} \mathrm{y} \mathrm{Y}_{\mathrm{f}}=\mathrm{Y}_{\max }$, obtenidos a partir de la incidencia acumulada $(\mathrm{p}=0.05)$ (Cuadro 1). Adicionalmente, no se detectaron interacciones significativas entre estos tratamientos y las parcelas grandes con y sin barreras, lo cual sugiere que los efectos positivos de cada uno de los componentes agronómicos del MI son aditivos y enfatizan la ausencia de diferencias entre los tratamientos MI-A y MI-D. El efecto positivo de las barreras vegetales en retrasar el inicio y progreso de las epidemias, no se reflejó en la reducción de la severidad final $\left(\mathrm{S}_{\mathrm{f}}\right),(\mathrm{p}=0.05)$. Esto puede explicarse por la interferencia negativa de las barreras vegetales sólo con la diseminación del virus entre plantas (medida a través de la incidencia) y no con el proceso de patogénesis (medido a través de la severidad) una vez que ha ocurrido una transmisión exitosa. Por lo anterior, la incidencia se constituye como la medida de intensidad de enfermedad apropiada para evaluar el efecto de barreras dentro de una técnica de manejo integrado. Por otro lado, resultan necesarios estudios futuros para entender la sinergia entre los diversos factores que integran esta técnica de manejo integrado.

\section{Efecto de tratamientos en vigor y producción}

El inicio de floración en los distintos tratamientos fue en promedio, de 78 DDT. La altura al primer fruto amarrado fue de 50 cm, lo cual coincidió con Mirafuentes-Hernández (10). No se encontraron diferencias estadísticas en altura de plantas y diámetro de tallo en parcelas con y sin barreras, pero sí en el tratamiento MI-A, asociado a parcelas chicas, respecto al TT (Tukey, $\mathrm{p}=$ 0.05). (Cuadro 2). Es posible que la ausencia de diferencias estadísticas en los otros tratamientos correspondientes a parcelas chicas se haya debido al efecto fitotóxico de la citrolina, tal y como se observa al comparar los valores obtenidos en estos parámetros en MI-A (Diámetro=7.7 $\mathrm{cm}$ y altura final de planta $=1.3 \mathrm{~m}$ ), respecto a MI (7.4 cm y $1.3 \mathrm{~m})$ y MI-D $(7.2 \mathrm{~cm}$ y $1.3 \mathrm{~m}) . \quad \mathrm{La}$ fitotóxicidad debida a la acumulación de citrolina, combinada con altas temperaturas $\left(>38^{\circ} \mathrm{C}\right)$ que se presentaron durante el experimento, causó quemaduras extensivas en la planta y defoliación prematura en las hojas de las plantas en MI y MI-D. Hernández-Castro (7) obtuvo estos mismos resultados con 
aplicación de citrolina en Veracruz. El uso de aceites minerales es promisorio en el control de enfermedades virales transmitidas en forma no persistente. Diversas hipótesis explican el modo de interferencia de los aceites sobre la transmisión de virus por áfidos: a) el aceite modifica la fisiología general de la planta, b) provoca disturbios en las células inoculadas, y 3) interfiere con el acoplamiento e incremento de partículas virales en el estilete de los áfidos Harris y Maramosch, (1977); Vanderveken, (1977) y Mosqueda et al., (1990) $(6,25,16)$ evaluaron aceites vegetales y minerales asperjados en diferentes concentraciones y concluyeron que la citrolina al $2 \%$ retrasó la aparición del PRSV-P. Estos investigadores y Galindo et al. (1978) (4) sugirieron continuar en esta línea mediante el ajuste de la técnica para mejorar su eficiencia y evitar quemaduras de flores, follaje y frutos de papayo. Una opción es ensayar aceites minerales con mayor grado de refinación para evitar toxicidad como el aceite isoparafínico de petróleo SafeT-Side ${ }^{\circledR}$ usado por Valera (24) en 2003 sin causar problemas de fitotoxicidad en papayo. Así mismo, conviene evaluar frecuencias de aplicación en concordancia con las tasas de emisión foliar ajustada para cada variedad y región.

No se observaron diferencias en el rendimiento entre parcelas grandes con y sin barreras pero sí en el tratamiento MI, asociado a parcelas chicas, respecto al TT (Tukey p d" 0.05) (Cuadro 2). El rendimiento para la variedad Maradol a una densidad de 2222 plantas ha ${ }^{-1}$ fluctúa entre 30 y 60 ton ha $^{-1}$ y depende del manejo del cultivo (10), mientras que con una densidad de 1100-1320 plantas $\mathrm{ha}^{-1}$ se obtiene un rendimiento de 63 ton ha ${ }^{-1}$ (9). Estos resultados muestran que aún cuando se ha experimentado con diferentes densidades de plantación, no se ha definido la densidad óptima, la cual puede variar dependiendo de las condiciones edáficas y climáticas y de los niveles de tecnología aplicada en una determinada región. En Veracruz, se encontró que el tipo Cera tuvo el mayor rendimiento en densidades altas (4400 plantas ha ${ }^{-1}$ ), con respecto a la tradicional (1111 plantas $\left.\mathrm{ha}^{-1}\right)$, sin favorecer el incremento de las tasas epidémicas del PRSV-P (18), debido a que el incremento de la incidencia de la enfermedad no depende del espaciamiento de plantas, pero sí de la densidad, estacionalidad y movimiento de los insectos vectores, los cuales pueden exhibir un patrón de dispersión aleatorio y/o uniforme (13). Adicionalmente, a mayores densidades, se compensa la pérdida de plantas por la práctica de eliminación de plantas por enfermedad en la técnica MI. Por lo anterior, en Michoacán, se justifica evaluar densidades mayores a la tradicional (20). Para la estimación de la producción (P) in situ se generaron dos modelos de regresión lineal simple a partir de la medición del semiperímetro y diámetro: a) con barreras: $\mathrm{P}$ $\left.=1.3909 \mathrm{x}-194.83, \mathrm{r}^{2}=0.8382 ; \mathrm{b}\right)$ sin barreras: $\mathrm{P}=1.676 \mathrm{x}-443.35, \mathrm{r}^{2}$ $=0.9302$. Un modelo general se propone con el promedio de los parámetros respectivos: $\mathrm{P}=1.53345 \mathrm{x}-319.09, \mathrm{r}^{2}=0.8842$. Es deseable que en estudios futuros, la estimación in situ de producción de papaya cv. Maradol sea una etapa sencilla que permita obtener resultados de forma rápida y confiable en las distintas fechas de corte.

En resumen, el progreso temporal de la enfermedad de las diferentes epidemias registradas en Michoacán fue similar a la observada en la región central norte de Veracruz. Las epidemias fueron de tipo sigmoidal, adecuadamante descritas por el modelo de Weibull y contrastaron en los valores alcanzados en $\mathrm{X}_{0}, \mathrm{Y}_{\mathrm{f}}, \mathrm{y} \mathrm{T}_{\mathrm{f}}$ e intensidad $\left(\mathrm{b}^{-1}\right)$. Se valida positivamente el uso comercial de barreras vegetales de jamaica, sembradas al menos 20 días antes del transplante, pero combinada con la eliminación temprana de plantas virosas como componentes de la técnica MI para mejorar la sanidad y rendimiento de plantaciones de papayo cv. Maradol en Michoacán, México. La citrolina combinada con altas temperaturas resultó fitotóxica y afectó el vigor de las plantas $(5.3 \%)$.

\section{AGRADECIMIENTOS}

A productores cooperantes y técnicos: Tiburcio Mendoza, Eleazar Peñalosa, Dagoberto Guillén, Francisco Marroquín, Francisco Durán, Rebeca Peña, Graciela Ávila, Prometeo Sánchez, Juan A. Villanueva y Carlos Acosta $\dagger$. A Sami Michereff (UFRPE, PE, Brasil) por la revisión técnica del escrito. Al CONACYT (19980301016) y el Colegio de Postgraduados por el financiado otorgado.

\section{REFERÊNCIAS BIBLIOGRÁFICAS}

1. Aguilar-Ruiz, G.; Ávila-Quezada, G.; Barrios-Díaz, B.; ChavarínPalacios, J.; Cristobal-Alejo, J.; Gonzalez-Medina, R.; GutierrezAyala, H.; Hernandez-Martínez, M.; Higuera-Salazar, V.; JarabaNavas, J.; Monsiváis-Loza, Y.; Mora-Aguilera, G.; Muñoz-Rodriguez, R.; Ramirez-Suarez, A.; Rivas-Valencia, P.; Romero-Hijo, R.; Tovar-Soto, A. Patrones espaciales en la incidencia de la mancha anular y su efecto en el tamaño y esquemas de muestreo. In: Congreso Latinoamericano de Fitopatología, 10., 1999, Guadalajara, México. Memorias ... Ciudad de México: Sociedad Mexicana de Fitopatología, 1999. p.286.

2. Becerra-Leor, E. Manejo integrado del virus de la mancha anular del papayo. Revista de la Universidad Cristóbal Colón, Veracruz, v.11, n.1, p.17-27, 1994.

3. Becerra-Leor, E. Preferencia al color del papayo (Carica papaya L.) y barreras de jamaica (Hibiscus sabdariffa L.) como medios para reducir transmisión por áfidos de virosis en papayo. Revista Mexicana de Fitopatología, México, v.7, n.3, p.218-222, 1989.

4. Galindo, A. J., Olivas, E. y Rodríguez, M. R. Experimento para controlar el virus de la Mancha Anular del Papayo. In: Congreso Nacional de Fitopatología. Oaxtepec, Morelos, México: 1978. Memorias... Ciudad de México: Sociedad Mexicana de Fitopatología.1978. p. 44-45.

5. Grupo Interdisciplinario e Interinstitucional de Investigación en Papayo. La mancha anular del papayo en Veracruz, México y su manejo integrado. In: Reunión Científica del Sector Agropecuario y Forestal del Estado de Veracruz, 7., 1994, Veracruz, México. Memórias ... Ciudad de Veracruz: Secretaría de Agricultura y Recursos Hidraúlicos/Instituto Nacional de Investigaciones Forestales, Agrícolas y Pecuarias/Universidad Veracruzana/Colégio de Postgraduados/Universidad Nacional Aautónoma de México/ Universidad Autônoma de Chapingo, 1994. p.87-92.

6. Harris, F. K. and Maramorosch, K. Aphids as Virus Vector. Academic Press. New York. USA. 1977. 559 p.

7. Hernández-Castro, E. Comportamiento del virus de la mancha anular del papayo bajo tres sistemas de manejo en el cv. "Maradol roja", en el Mpio. de Paso de Ovejas, Veracruz. 1998. 83 f. Tesis (Maestría en Ciencias) - Campus Veracruz, Colegio de Postgraduados, Veracruz.

8. Instituto Nacional de Estadística, Geografía y Informática. Sistemas nacionales estadístico y de información geográfica. México: INEGI, 2005. Disponivel em: <http://www.inegi.gob.mx/ geo>. Acesso em: 15 jan. 2005.

9. Mandujano-Barrios, R. El papayo. Tuxpan: Universidad Veracruzana, 1993. 37p. (Publicación Tecnica, 1).

10. Mirafuentes-Hernández, F. Manual para la producción de papayo en Tabasco. Tabasco: Instituto Nacional de Investigaciones Forestales y Agropecuarias - Campo Experimental Huimanguillo, 1997. 24 p. (Folleto Técnico, 9).

11. Mora-Aguilera, G.; Gutiérrez-Espinoza, A.; Téliz-Ortiz, D. Recursos agrícolas del trópico y subtrópico Mexicano. Montecillo: Instituto Nacional de Estadística y Geografía / Colegio de Postgraduados, 1998. 174p.

12. Mora-Aguilera, G.; Nieto-Angel, D.; Campbell, C.L.; Teliz-Ortiz, 
D.; Garcia-Perez, E. Multivariate comparison of papaya ringspot epidemics. Phythopathology, St.Paul, v.86, n.1, p.70-78, 1996.

13. Mora-Aguilera, G.; Nieto-Angel, D.; Téliz-Ortiz, D.; Campbell, C.L. Development of a prediction model for papaya ringspot in Veracruz, Mexico. Plant Disease, St. Paul, v.77, n.12, p.1205$1211,1993$.

14. Mora-Aguilera, G.; Téliz-Ortiz, D.; Campbell, C.L.; Avila-Catarino R. Temporal and spatial development of papaya ringspot in Veracruz, Mexico. Journal of Phythopathology, Berlin, v.136, n.1, p.27-36, 1992 .

15. Morshidi, B.M.; Manshardat, R.M. Where did papayas come from? In: Hawaii agriculture: positioning for growth. Manoa: University of Hawaii at Manoa, 1995. p.108-109.

16. Mosqueda, V. R., Becerra, L. N. E. y De los Santos, de la R. F. Aplicación de aceites en papayo para retrasar la epidemia del virus de la mancha anular del papayo. In: XVIII Congreso Nacional de la Sociedad Mexicana de Fitopatología. Culiacán, México. Memorias. Sociedad Mexicana de Fitopatología. 1990. p. 93.

17. Nelson, M.R.; Tutlttle, D.M. The epidemiology of cucumber mosaic and watermelon mosaic 2 of cantalaups in an arid climate. Phytopathology, St. Paul, v.59, n.8, p.849-856, 1969.

18. Nieto-Angel, D. Epidemiología del virus de la mancha anular del papayo bajo diferentes fechas de siembra, densidades de plantación y localidades en Veracruz. 1990. $68 \mathrm{f}$ Tesis (Maestría en Ciencias) - Instituto de Fitosanidad-Colegio de Postgraduados, Montecillo, México.

19. Pennypaker, S.P.; Knoble, H.D.; Antle, C.D.; Madden, L.V. A flexible model for studying plant disease progression. Phyto- pathology, St. Paul, v.70, n.3, p.232-235, 1980.

20. Rivas-Valencia, P.; Mora-Aguilera, G.; Teliz-Ortiz, D.; MoraAguilera, A.; Acosta-Zamudio, C. Respuesta de cuatro variedades de papayo bajo un sistema de manejo integrado. In: Congreso Nacional de la Sociedad Mexicana de Fitopatología. 2000. Puerto Vallarta, Jalisco. Memorias. Sociedad Mexicana de Fitopatología, 2000. p.64.

21. SAS, Institute, INC. Use's guide: Statistics. Release 6.03 Ed. SAS Institute, INC. Cary, N. C. USA., 1988. 1028 p.

22. Secretaria de Agricultura, Ganadería, Desarrollo Rural, Pesca y Alimentación. Sistema integral de información agroalimentaria y pesquera. Ciudad de México: SAGARPA, 2005. Disponivel em: <http://www.siap.sagarpa.gob.mx/ ar_comagri.html>. Acesso em: 22 dec. 2008.

23. Téliz-Ortiz, D.; Mora-Aguilera, A.; Nieto-Angel, D.; Gonsalves, D.; García-Pérez, E.; Matheis-Toledano, L.; Ávila-Resendiz, C. Mancha anular del papayo en México. Revista Mexicana de Fitopatología, México, v.9, n.1, p.64-68, 1991.

24. Valera Jardines Félix. Aceites minerales, composición y abundancia de áfidos y sus relaciones con el progreso epidemiológico del PRSV-P en una plantación de papayo (Carica papaya L.) en Michoacán, México. 2003. 46 f. Tesis (Maestría en Ciencias) - Instituto de FitosanidadColegio de Postgraduados, Montecillo, México.

25. Vanderveken, J. J. Oils and other inhibitors of nopersistent virus transmission. In: Harris, F. K. And Maramorosch, K(eds.). Aphids as Virus Vector. Academic Press. New York. USA. 1977. p. 435-454. 